\title{
Two-mode interference measurement for nanometer accuracy absolute ranging
}

\author{
Duy-Ha Phung, ${ }^{1}$ Christophe Alexandre, ${ }^{2}$ and Michel Lintz ${ }^{1, *}$ \\ ${ }^{1}$ ARTEMIS, Université de Nice Sophia, Observatoire de la Cote d'Azur, and CNRS, Bd de l'Observatoire, Nice 06300, France \\ ${ }^{2}$ CEDRIC/LAETITIA, CNAM, 292 Rue Saint Martin, 75141 Paris Cedex 03, France \\ *Corresponding author: michel.lintz@oca.eu
}

Received October 9, 2012; revised December 7, 2012; accepted December 17, 2012;

posted December 20, 2012 (Doc. ID 177487); published January 17, 2013

\begin{abstract}
We demonstrate a laser ranging scheme that uses a high-frequency modulated beam to achieve subnanometer precision by the combined use of interferometric and time-of-flight measurements. We first describe how the absolute distance is extracted from a two-mode interference signal. In particular, we show that the signal, which presents both optical and synthetic wavelength scales, allows one to achieve nanometer-scale accuracy, despite the significant long-term phase drifts in the $20 \mathrm{GHz}$ detection chains. We present results obtained with the telemeter implemented for a distance of about four meters, obtained by folding the laser beam path to the target. () 2013 Optical Society of America

OCIS codes: $280.3400,260.3160,120.5050,120.6085$.
\end{abstract}

In the electronic measurement of long distances, multiwavelength interferometry has been able to provide both long synthetic wavelength and sub-micron accuracy [1] Using multiple $40 \mathrm{GHz}$ phase modulations of a CW laser, MSTAR [2] has reached nm-scale accuracy. Frequency combs from femtosecond lasers are increasingly used in absolute distance measurement setups inspired either from optical linear sampling [3] , or from dispersive interferometry [4].

A different approach combines

(i) a high precision, interferometric optical phase measurement, wrapped in the $\left[0, \lambda_{\text {opt }}\right]$ interval;

(ii) a carrier modulation phase measurement at frequency $F \approx 20 \mathrm{GHz}$, wrapped in the $[0, \Lambda \equiv c / F]$ interval; and

(iii) a time-of-flight measurement of the distance $d$.

If measurement (iii) is accurate enough to bring unambiguous meaning to measurement (ii), which in turn has good enough accuracy to give absolute significance to measurement (i), then absolute distances can be measured with an accuracy far better than the optical wavelength. It appears, however, that long-term phase drifts in high-frequency measurements exceed the limit $\pi \lambda_{\text {opt }} / \Lambda$ beyond which measurement (ii) cannot provide unambiguous determination of $2 d / \lambda_{\text {opt }}$.

Here we demonstrate that this problem is solved by a measurement scheme in which both phase measurements (i) and (ii) are obtained consistently from the same interference measurement, using a $20 \mathrm{GHz}$ beatnote, two-mode laser beam. In a simple setup on which a $\approx 7.5 \mathrm{~m}$ optical path length is implemented, we show that this measurement scheme can achieve the conditions required to provide nanometer accuracy absolute distance measurements. In this Letter we present the two-mode interference signal, its properties, and its exploitation for nm-scale accuracy absolute distance measurement.

Two narrow-linewidth, single-mode fiber lasers at $1.54 \mu \mathrm{m}$ are phase-locked ([5], Section 5) at a fixed frequency of $F=20.04 \mathrm{GHz}$. The corresponding synthetic wavelength $\Lambda=c / F$ is close to $15 \mathrm{~mm}$. The two-mode $\left[\nu_{\mathrm{opt}}, \nu_{\mathrm{opt}}+F\right]$ laser beam propagates, from BS0 to PhD1 in Fig. 1 below, along two different paths: a reference path of constant length $l$, and a variable measurement path of length $L$. The interference of these two beams provides the signal from which we extract the quantity to be measured: the length difference between the two paths: $\Delta L \equiv L-l$.

In Fig. 1, we assume that the polarization of the initial beam is oriented so that a small fraction of the power, $\varepsilon^{2}=\mathrm{a}$ few $\%$, propagates from PBS to BS, along the reference path with $S$ polarization. The remaining power (fraction $1-\varepsilon^{2}$ close to $100 \%$ ) propagates along the measurement path with $P$ polarization, from PBS, via the target, to the half-wave plate $(S$ polarization after the half-wave plate) and interferes with the reference beam. The resulting intensity,

$$
\begin{aligned}
I(t)= & \left(1-\varepsilon^{2}\right)[1+\cos (\delta(t-L / c))]+\varepsilon^{2}[1+\cos (\delta(t-l / c))] \\
& +2 \varepsilon \sqrt{1-\varepsilon^{2}} \cos (\omega(L-l) / c+\delta(L-l) / 2 c) \\
& \times[\cos (\delta(t-(L+l) / 2 c))+\cos (\delta(L-l) / 2 c)],
\end{aligned}
$$

where $\omega \equiv 2 \pi \nu_{\text {opt }}=2 \pi c / \lambda_{\text {opt }}, \delta \equiv 2 \pi F=2 \pi c / \Lambda$, is detected by photodiode PhD1. The (reference) photodiode $\mathrm{PhD0}$ delivers a signal in which amplitude and phase are used to eliminate power and phase fluctuations of the laser sources. Expression (1) is valid only in a vacuum. Application to quantitative measurements in air would require two indices of refraction, at the two wavelengths, to be taken into account.

The measurement exploits the phase and amplitude of the $\cos (\delta t)$ terms, oscillating at the high frequency $F$. From Eq. (1), by using the vector representation in the complex plane, we obtain the measurement signal vector $\boldsymbol{a}$ :

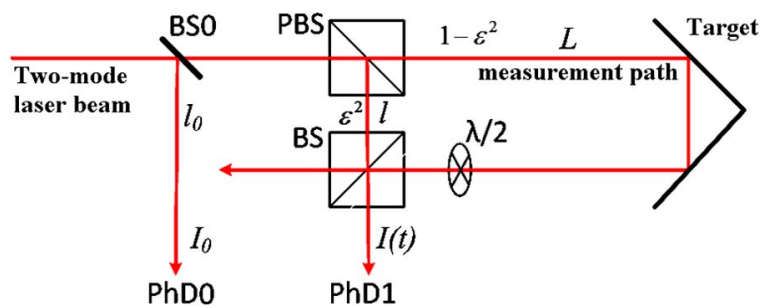

Fig. 1. (Color online) Schematic diagram of the telemeter. BS0, beam splitter plate; (P)BS, (polarizing) beam splitter cube. 


$$
a=\text { meas }+ \text { ref }+ \text { Int, }
$$

where meas $=\left(1-\varepsilon^{2}\right) e^{i \delta L / c}$, ref $=\varepsilon^{2} e^{i \delta l / c}$, and

$$
\text { Int }=2 \varepsilon \sqrt{\left(1-\varepsilon^{2}\right)} \cos ((\omega+\delta / 2) \Delta L / c) e^{i \delta(L+l) / 2 c} .
$$

The vector Int is the interference contribution. When the optical frequency $\nu_{\text {opt }}$ of the two-mode source varies over more than the free spectral range FSR $\equiv c / \Delta L$, the tip of the vector $a$ travels back and forth along the "segment" [Fig. 2(a)], of length $4 \varepsilon \sqrt{1-\varepsilon^{2}}$, characteristic of the optical interference. Any deviation from a strictly linear segment reveals the presence of systematic effects.

The dependence on the target position is more complex. Not only the amplitude of the Int vector changes, but its direction and the direction of the meas vector also change. The pattern obtained when the target is moved by half a synthetic wavelength displays a large number $\left(\omega / \delta \approx 10^{4}\right)$ of spikes. The spikes are illustrated in Fig. 2(b), with the choice of $\omega / \delta \approx 20$ for the spikes to be conveniently observable. Each spike corresponds to one fringe of the optical interference.

From a single measurement of this complex interference signal (one single data point in the complex plane) one cannot extract the three contributions separately. However, assuming that a time-of-flight measurement has been done beforehand with a relative accuracy of $\approx 10^{-5}$ or better, one has an estimate of the free spectral range FSR. Then, one can perform three measurements$a 1, a 2, a 3-w h e r e$, using an acousto-optic modulator (AOM), the optical frequency of the beam is changed from $\nu_{\text {opt }}$ to $\nu_{\text {opt }}+$ FSR/4 and then to $\nu_{\text {opt }}+$ FSR/2, respectively. Then these three data points of the two-mode interference signal can be processed in a relatively straightforward manner to compute, separately, the interferometric phase $\Phi_{\text {inter }} \equiv 2 \pi \Delta L / \lambda_{\text {opt }}$ (modulo $2 \pi$ ), and synthetic phase $\Phi_{\text {synt }} \equiv 2 \pi \Delta L / \Lambda$ (modulo $2 \pi$ ).

We perform a measurement of $a$ every $15 \mu$ s and assume that $\Delta L$ does not vary during the three-state pattern. With this three-fold data group - $a 1, a 2, a 3$ - we first calculate the middle, the length, and the direction of the segment. And since $\varepsilon^{2} \ll 1$, we can use a perturbative treatment to subtract the ref contribution and obtain the meas vector. Ideally, one can use the orientation of the meas signal in the complex plane to obtain the value of the synthetic phase. In practice, this approach will fail, due to the slow drifts (larger than $10^{-4}$ cycle at the $\approx 1$ min time scale, [Fig. 4]) in the measurement chains. However, the direction of the segment [Eq. (3)] provides another determination of the synthetic phase, and the combination
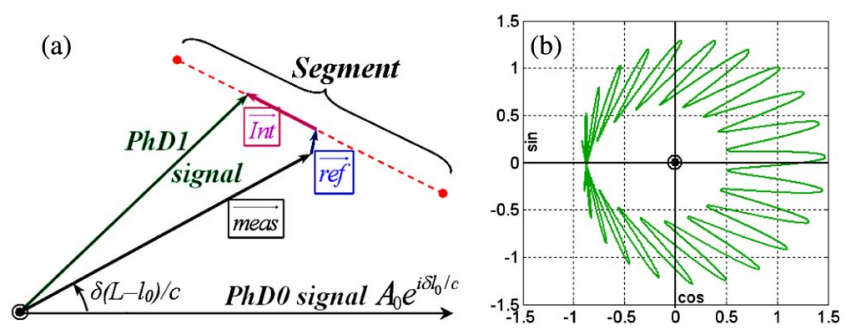

Fig. 2. (Color online) Signal behavior: (a) when the optical frequency varies and (b) when the target is moved continuously over $\Lambda / 2$, with $\varepsilon \approx 0.25$. of these two provides $(L-l) / \Lambda$ in a way which eliminates the slow drifts. This point can be seen by writing $(L-l) / 2 \Lambda=\left(L-l_{0}\right) / \Lambda-\left(L+l-2 l_{0}\right) / 2 \Lambda$, where $l_{0}$ is the path length on the PhD0 detection chain. Finally, we calculate the interferometric phase.

For the procedure to be applicable, it has to fulfil two conditions. First, the three-fold pattern must be completed in a time short enough so that $\Delta L$ can be considered as static during the $3 \times(15 \mu \mathrm{s})$ data taking. This leads to a limit of $2.6 \mu \mathrm{m} / \mathrm{s}$ to the target speed. This limit is unrealistic. A modified, yet straightforward, procedure with five points-a $1, \boldsymbol{a} 1, \boldsymbol{a} 2, \boldsymbol{a} 3, \boldsymbol{a} 3$ - supports $300 \mu \mathrm{m} / \mathrm{s}$ target speeds. Second, the accuracy of the phase and of the amplitude ratio measurements should be respectively much better than $10^{-4}$ cycle and $10^{-4}$; otherwise the procedure may converge to a value of $\Delta L$ shifted by integer numbers of the optical wavelength $\lambda_{\text {opt }}$. This sets upper limits to systematic errors, either with optical origin (optical cross talk or stray interference, presence of sidebands on the two-mode laser source spectrum), or due to electronic imperfections (cross talk in the electronic measurement channels, amplitude-to-phase coupling []]). Similarly, the statistical noise on one elementary cycle of the procedure (a five-fold data group) is not good enough. To achieve the required statistical precision, $N=$ 320 measurements of five-fold data groups are necessary. The ranging procedure supports the movement of the target and allows us to average $N$ five-fold data groups, yet keeping the benefit of interferometric measurement.

In the experimental setup (Fig. 3), interference takes place at the Glan polarizer which, oriented at $45^{\circ}$, mixes the beams from the reference and measurement arms. Parallel optics, such as PBS, BS cubes, or plates, have been replaced by wedged optics to eliminate multiple reflexions.

The microwave signals $(20.04 \mathrm{GHz})$ are detected by two $20 \mathrm{GHz}$ bandwidth photodiodes, down-converted to $20 \mathrm{MHz}$ by the mixers and sampled during $10 \mu \mathrm{s}$ by two $250 \mathrm{MS} / \mathrm{s}, 14$ bit analog-to-digital converters. The phase meter updates the $\mathrm{AOM}$ frequency $f_{\mathrm{AOM}}$ for the frequency shifts and calculates the signal amplitudes and phases using the CORDIC algorithm [7]. The AOM allows

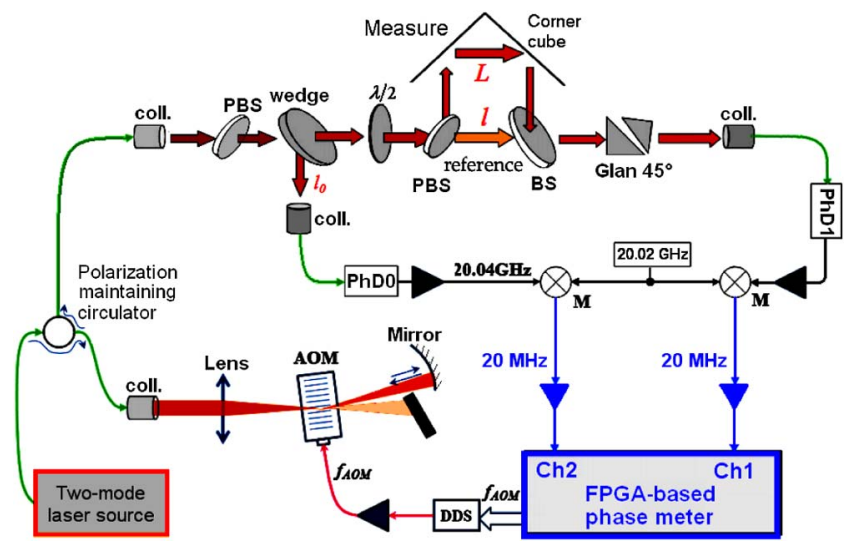

Fig. 3. (Color online) Schematic diagram of the telemeter. Orange, laser beams; green, optical fiber; black, HF signals (20.04 GHz); Blue, RF signals (20 MHz); coll., fibered collimator; (P)BS, (polarizing) beam splitter; wedge, wedged splitter plate; Glan $45^{\circ}$, air Glan polarizer, oriented at $45^{\circ}$; AOM, acousto-optic modulator, range of $f_{\mathrm{AOM}}$ is [135-185] MHz; DDS, direct digital synthesizer; M, mixers; FPGA, field programmable gate array. 


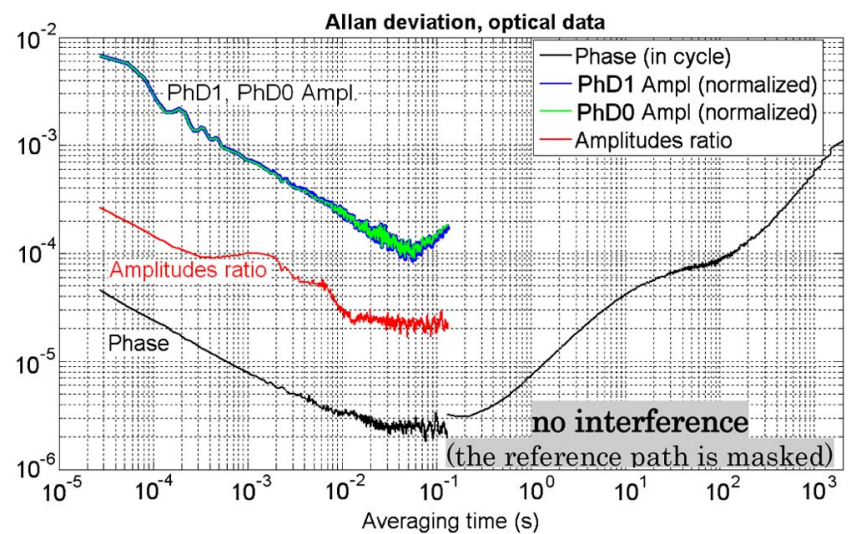

Fig. 4. (Color online) Stability of the relative phase and amplitude ratio measurements with a stable optical signal without interference.

for a $200 \mathrm{MHz}$ maximum FSR, hence a minimum length difference of $\Delta L \approx 1.5 \mathrm{~m}$ ( $0.75 \mathrm{~m}$ distance).

In Fig. 4 , noise on the amplitude and phase of optical signals is plotted as Allan deviation curves. It exceeds the phase meter noise floor.

A full cycle of the measurement procedure (1600 data points, or $N=320$ five-fold data groups) corresponds to $43 \mathrm{~ms}$ of data taking. With the observed noise (Fig. 4), simulations predict $96 \%$ of convergence of the ranging results. At longer time scales ( $1 \mathrm{~s}$ and above) phase stability degrades, reaching $10^{-3}$ cycle at $30 \mathrm{mn}$.

The ranging procedure is used to measure $\mathrm{a} \sim 3.75 \mathrm{~m}$ distance (FSR $\approx 40 \mathrm{MHz}$ ). Figure $\underline{5(\mathrm{a})}$ shows the 320 results of 320 elementary measurement cycles, revealing nm-scale acoustic perturbations of the $7.5 \mathrm{~m}$ path. Figure 5(b) shows the instrumental noise (the reference path is masked, so that no interference takes place).

From the noise observed on the data without interference one can expect a precision of around $100 \mathrm{pm}$ on each elementary five-fold measurement cycle (duration $\approx 100 \mu \mathrm{s}$ ), and less than $10 \mathrm{pm}$ after the full cycle of a ranging measurement (43 ms data).

Figure 6 shows the results of 5000 complete measurement cycles while the target moves slowly at about

\section{Stability of the length measurement at short time scales}
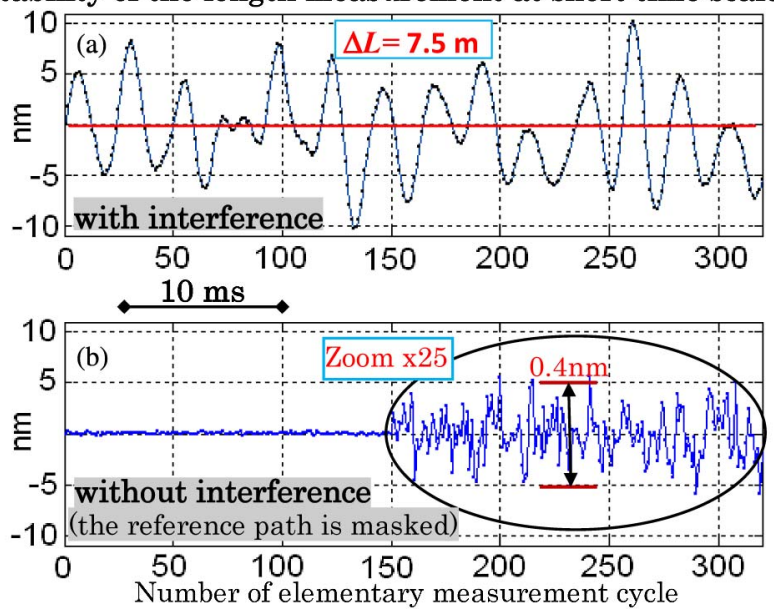

Fig. 5. (Color online) (a) Stability of the measurement with 320 elementary cycles in $43 \mathrm{~ms}$ and (b) signal noise, converted into length data.
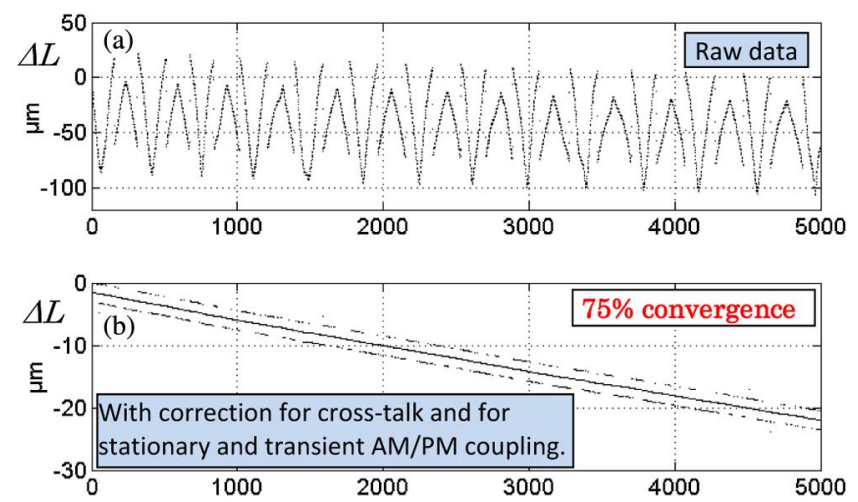

Fig. 6. (Color online) Results of 5000 full cycles (a) without and (b) with correction for electronic distortion. For convenience, the length values are shifted by $\approx 7.5 \mathrm{~m}$.

$2 \mu \mathrm{m} / \mathrm{min}$ during $5 \mathrm{~min}$. If no correction is made [Fig. 6(a)], systematic errors are observed, up to $\pm 50 \mu \mathrm{m}$.

After correcting for cross talk and AM-to-PM coupling, the length values, as shown in Fig. $\underline{6(b)}$, correspond to the regular, continuous target motion. The residual errors (values shifted by $\pm \lambda_{\text {opt }}$ ) are not randomly scattered, and are likely to be due to imperfect correction of the time-dependent AM-to-PM coupling []]. Indeed, by choosing a different timing for the data acquisition, convergence rates of $94 \%$ have been reached. To approach $100 \%$ convergence, further work should focus on electronic imperfections.

With these preliminary results we demonstrate that, on a relatively simple setup, the two-mode interference signal can be exploited for high-precision, high-accuracy absolute distance measurement, if combined with a time-of-flight measurement. From the observed noise on the two-mode interference signal, and assuming negligible master laser frequency noise, one can expect a precision of about $6 \mathrm{pm}$ on a $43 \mathrm{~ms}$ signal data set. The measurement update rate is currently $\approx 60 \mathrm{~ms}$ but, at least in principle, nothing prevents from achieving an update every 100 or $200 \mu \mathrm{s}$ with a precision of about $100 \mathrm{pm}$, provided commercial programmable circuits are powerful enough to complete the required data processing.

This work was supported by L'Agence Nationale de la Recherche (ANR), Paris, (Grant ANR-07-BLAN-0309-01) and the Centre national d'études spatiales (CNES), Paris.

\section{References}

1. Y. Salvadé, N. Schuhler, S. Lévêque, and S. Le Floch, Appl. Opt. 47, 2715 (2008).

2. O. P. Lay, S. Dubovitsky, R. D. Peters, J. P. Burger, S.-W. Ahn, W. H. Steier, H. R. Fetterman, and Y. Chang, Opt. Lett. 28, 890 (2003).

3. I. Coddington, W. C. Swann, L. Nenadovic, and N. R. Newbury, Nat. Photonics 3, 351 (2009).

4. S. A. van den Berg, S. T. Persijn, G. J. P. Kok, M. G. Zeitouny, and N. Bhattacharya, Phys. Rev. Lett. 108, 183901 (2012).

5. J. Fatome, S. Pitois, C. Fortier, B. Kibler, C. Finot, G. Millot, C. Courde, M. Lintz, and E. Samain, Opt. Commun. 283 2425 (2010).

6. D. H. Phung, M. Lintz, and C. Alexandre, "Dynamics of the amplitude-to-phase coupling in $1.5 \mu \mathrm{m}$ high bandwidth photodiodes," IEEE Trans. Microwave Theor. Tech., submitted for publication.

7. J. E. Volder, IRE Trans. Electron. Comput. 8, 330 (1959). 